\title{
Fuzzy rg-Super Irresolute Mapping
}

\author{
${ }^{1}$ M. K. Mishra ${ }^{2}$ Manisha Shukla, ${ }^{3}$ R. Lakshmirekha \\ ${ }^{1}$ Professor, ${ }^{2}$ Asst. Prof.AGCW Karaikal, ${ }^{1,3}$ Asst Prof, EGS PEC Nagapattinam
}

\begin{abstract}
In this paper the concept of fuzzy rg -super irresolute mappings have been introduced and explore some of its basic properties in fuzzy Topological Space.
\end{abstract}

Keywords: fuzzy topology, fuzzy super closure, Fuzzy Super Interior fuzzy rg-super closed sets and fuzzy rgsuper open sets, fuzzy rg-super continuous and fuzzy rg -super irresolute mappings.

\section{Preliminaries}

Let $\mathrm{X}$ be a non empty set and $\mathrm{I}=[0,1]$. A fuzzy set on $\mathrm{X}$ is a mapping from $\mathrm{X}$ in to $\mathrm{I}$. The null fuzzy set 0 is the mapping from $X$ in to I which assumes only the value is 0 and whole fuzzy sets 1 is a mapping from $X$ on to I which takes the values 1 only. The union (resp. intersection) of a family $\left\{A_{\alpha}: \alpha \in \Lambda\right\}$ of fuzzy sets of $X$ is defined by to be the mapping sup $\mathrm{A}_{\alpha}$ (resp. inf $\mathrm{A}_{\alpha}$ ). A fuzzy set $\mathrm{A}$ of $\mathrm{X}$ is contained in a fuzzy set $\mathrm{B}$ of $\mathrm{X}$ if $\mathrm{A}(\mathrm{x}) \leq \mathrm{B}(\mathrm{x})$ for each $\mathrm{x} \in \mathrm{X}$. A fuzzy point $\mathrm{x}_{\beta}$ in $\mathrm{X}$ is a fuzzy set defined by $\mathrm{x}_{\beta}(\mathrm{y})=\beta$ for $\mathrm{y}=\mathrm{x}$ and $\mathrm{x}(\mathrm{y})=0$ for $\mathrm{y} \neq \mathrm{x}$, $\beta \in[0,1]$ and $y \in X$.A fuzzy point $x_{\beta}$ is said to be quasi-coincident with the fuzzy set $A$ denoted by $x_{\beta q} A$ if and only if $\beta+A(x)>1$. A fuzzy set $A$ is quasi coincident with a fuzzy set $B$ denoted by $A_{q} B$ if and only if there exists a point $\mathrm{x} \in \mathrm{X}$ such that $\mathrm{A}(\mathrm{x})+\mathrm{B}(\mathrm{x})>1 . \mathrm{A} \leq \mathrm{B}$ if and only if $\rceil\left(\mathrm{A}_{\mathrm{q}} \mathrm{B}^{\mathrm{c}}\right)$.A family $\tau$ of fuzzy sets of $\mathrm{X}$ is called a fuzzy topology [2] on $X$ if 0,1 belongs to $\tau$ and $\tau$ is super closed with respect to arbitrary union and finite intersection. The members of $\tau$ are called fuzzy super open sets and their complement are fuzzy super closed sets. For any fuzzy set A of $\mathrm{X}$ the closure of A (denoted by $\operatorname{cl}(\mathrm{A})$ ) is the intersection of all the fuzzy super closed super sets of A and the interior of A (denoted by int(A) )is the union of all fuzzy super open subsets of A.

Defination1.1 $[5,10,11,12]$ : Let $(X, \tau)$ fuzzy topological space and $A \leq X$ then

1. Fuzzy Super closure $\operatorname{scl}(\mathrm{A})=\{\mathrm{x} \in \mathrm{X}: \mathrm{cl}(\mathrm{U}) \cap \mathrm{A} \neq \phi\}$

2. Fuzzy Super interior $\operatorname{sint}(A)=\{\mathrm{x} \in \mathrm{X}: \mathrm{cl}(\mathrm{U}) \leq \mathrm{A} \neq \phi\}$

Definition 1.2[5, 10,11,12]: A fuzzy set A of a fuzzy topological space $(X, \tau)$ is called:

(a) Fuzzy super closed if scl(A) $\leq$ A.

(b) Fuzzy super open if 1-A is fuzzy super closed $\sin (\mathrm{A})=\mathrm{A}$

Remark 1.1[5, 10,11,12]: Every fuzzy closed set is fuzzy super closed but the converses may not be true.

Remark 1.2[5, 10,11,12]: Let A and B are two fuzzy super closed sets in a fuzzy topological space (X,I), then $A \cup B$ is fuzzy super closed.

Remark 1.3[5]: The intersection of two fuzzy super closed sets in a fuzzy topological space (X, I) may not be fuzzy super closed.

Definition 1.3: A fuzzy set A of an fuzzy topological space $(X, \mathfrak{I})$ is said to be :-

(a) fuzzy regular super open if $\mathrm{A}=\operatorname{int}(\mathrm{cl}(\mathrm{A}))$ [7].

(b) fuzzy g-super closed if $\mathrm{cl}(\mathrm{A}) \leq \mathrm{O}$ whenever $\mathrm{A} \leq \mathrm{O}$ and $\mathrm{O}$ is an fuzzy super open set.[14]

(c) fuzzy g-super open if $A^{c}$ is fuzzy g-closed.[14]

(d) fuzzy rg-super closed if $\mathrm{cl}(\mathrm{A}) \leq \mathrm{O}$ whenever $\mathrm{A} \leq \mathrm{O}$ and $\mathrm{O}$ is an fuzzy regular super open set.[16]

(e) fuzzy rg-super open if $\mathrm{A}^{\mathrm{c}}$ is fuzzy rg-closed.[16]

Remark 1.3: Every fuzzy super closed set is fuzzy g-super closed and every fuzzy g-super closed set is fuzzy rg-super closed but the converse may not be true.[14,16]

Definition 1.4: A mapping $\mathrm{f}:(\mathrm{X}, \mathfrak{J}) \rightarrow(\mathrm{Y}, \sigma)$ is said to be :

1. Fuzzy g-super continuous if the pre image of every fuzzy super closed set of $\mathrm{Y}$ is fuzzy g-super closed in X.[15].

2. Fuzzy rg-super continuous if the pre image of every fuzzy super closed set of $Y$ is fuzzy rg-super closed in X. [17]

Remark 1.4: Every fuzzy super continuous mapping is fuzzy g-super continuous and every fuzzy g-super continuous mapping is fuzzy rg-super continuous but the converse may not be true.[17]

Definition 1.5: A collection $\left\{G_{\alpha}: \alpha \in \wedge\right\}$ of fuzzy $r$-super open sets in a fuzzy topological space $(X, \mathfrak{I})$ is called a fuzzy rg-super open cover of an fuzzy set $A$ of $X$ if $A \leq \cup\left\{G_{\alpha}: \alpha \in \wedge\right\}$.[16]

Definition 1.6: A fuzzy topological space $(X, \mathfrak{J})$ is said to be fuzzy rg- super compact if every fuzzy rg-super open cover of $\mathrm{X}$ has a finite subcover.[16] 
Definition 1.7: An fuzzy set A of an fuzzy topological space (X, I) is said to be fuzzy rg- super compact relative to $X$ if every collection $\left\{G_{\alpha}: \alpha \in \wedge\right\}$ of fuzzy rg-super open subsets of $X$ such that $A \leq$ $\cup\left\{\mathrm{G}_{\alpha}: \alpha \in \wedge\right\}$ there exists a finite subset $\wedge_{0}$ such that $\mathrm{A} \leq \cup\left\{\mathrm{G}_{\alpha \mathrm{j}}: \alpha_{\mathrm{j}} \in \wedge_{0}\right\} .[16]$

Definition 1.8: A fuzzy topological space $X$ is fuzzy rg-connected if there is no proper fuzzy set of $X$ which is both fuzzy rg-super open and fuzzy rg-closed.[17]

\section{Fuzzy rg -super irresolute Mappings}

Definition 2.1: A mapping f from a fuzzy topological space $(X, \Im)$ to another fuzzy topological space $(Y, \sigma)$ is said to be fuzzy rg -super irresolute if the pre image of every fuzzy rg-super closed set of $\mathrm{Y}$ is fuzzy rg-super closed in X.

Theorem 2.1: A mapping $\mathrm{f}:(\mathrm{X}, \mathfrak{J}) \rightarrow(\mathrm{Y}, \sigma)$ is fuzzy $\mathrm{rg}$-super irresolute if and only if the pre image of every fuzzy $\mathrm{rg}$-super open set in $\mathrm{Y}$ is fuzzy $\mathrm{rg}$-super open in $\mathrm{X}$.

Proof: It is obvious because $\mathrm{f}^{-1}\left(\mathrm{U}^{\mathrm{c}}\right)=\left(\mathrm{f}^{-1}(\mathrm{U})\right)^{\mathrm{c}}$, for every fuzzy set $\mathrm{U}$ of $\mathrm{Y}$.

Remark 2.1: Every fuzzy g-super closed set is fuzzy rg-super closed it is clear that every fuzzy rg -super irresolute mapping is fuzzy rg-super continuous but the converse may not be true.

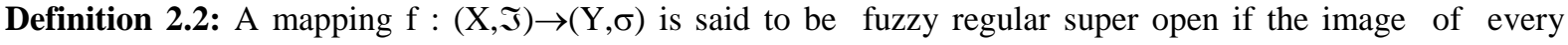
fuzzy regular super open set of $\mathrm{X}$ is fuzzy regular super open set in $\mathrm{Y}$.

Theorem 2.2: Let $\mathrm{f}:(\mathrm{X}, \mathfrak{I}) \rightarrow(\mathrm{Y}, \sigma)$ is bijective fuzzy regular super open and fuzzy rg-super continuous then $\mathrm{f}$ is fuzzy rg- super irresolute.

Proof: Let $A$ be a fuzzy rg-super closed set in $Y$ and let $f^{-1}(A) \leq G$ where $G$ is fuzzy regular super open set in $X$. Then $A \leq f(G)$. Since $f$ is fuzzy regular super open and $A$ is fuzzy rg-super closed in $Y, c l(A) \leq f(G)$ and $f^{-}$ ${ }^{1}(\mathrm{cl}(\mathrm{A})) \leq \mathrm{G}$. Since $\mathrm{f}$ is fuzzy rg-super continuous and $\mathrm{cl}(\mathrm{A})$ is fuzzy super closed in $\mathrm{Y}, \operatorname{cl}\left(\mathrm{f}^{-1}(\mathrm{cl}(\mathrm{A}))\right) \leq \mathrm{G}$. And so $\operatorname{cl}\left(\mathrm{f}^{-1}(\mathrm{~A})\right) \leq \mathrm{G}$. Therefore $\mathrm{f}^{-1}(\mathrm{~A})$ is fuzzy $\mathrm{rg}$-super closed in $\mathrm{X}$. Hence $\mathrm{f}$ is fuzzy rg-irresolute.

Theorem 2.3: Let $f:(X, \Im) \rightarrow(Y, \sigma)$ and $g:(Y, \sigma) \rightarrow(Z, \eta)$ be two fuzzy $r g$-super irresolute mappings, then gof : $(X, \Im) \rightarrow(Z, \eta)$ is fuzzy rg- super irresolute.

Proof : Obvious.

Theorem 2.4: Let $f:(X, \Im) \rightarrow(Y, \sigma)$ is fuzzy rg -super irresolute mapping, and if $B$ is fuzzy rg- super compact relative to $X$, then the image $f(B)$ is fuzzy rg- super compact relative to $Y$.

Proof : Let $\left\{A_{i}: i \in \wedge\right\}$ be any collection of fuzzy rg-super open set of $Y$ such that $f(B) \leq \cup\left\{A_{i}: i \in \wedge\right\}$. Then $B \leq$ $\cup\left\{f^{-1}\left(A_{i}\right): i \in \wedge\right\}$. By using the assumption, there exists a finite subset $\wedge_{0}$ of $\wedge$ such that $B \leq \cup\left\{f^{-1}\left(A_{i}\right): i \in \wedge_{0}\right\}$. Therefore, $f(B) \leq\left\{A_{i}: i \in \wedge_{0}\right\}$. Which shows that $f(B)$ is fuzzy rg- super compact relative to $Y$.

Theorem 2.5: A fuzzy rg -super irresolute image of a fuzzy rg- super compact space is fuzzy rg-compact.

Proof: Obvious.

Theorem 2.6: If the product space $(\mathrm{XxY}, \mathfrak{J x} \sigma)$ of two non- empty fuzzy topological spaces $(\mathrm{X}, \mathfrak{I})$ and $(\mathrm{Y}, \sigma)$ is fuzzy rg- super compact, then each factor space is fuzzy rg- super compact.

Proof: Obvious.

Theorem: 2.7:: Let $f:(X, \mathfrak{I}) \rightarrow(Y, \sigma)$ is a fuzzy rg -super irresolute surjection and $(\mathrm{X}, \mathfrak{I})$ is fuzzy rg- super connected, then $(\mathrm{Y}, \sigma)$ is fuzzy rg- super connected.

Proof : Suppose $\mathrm{Y}$ is not fuzzy rg- connected then there exists a proper fuzzy set $\mathrm{G}$ of $\mathrm{Y}$ which is both fuzzy rg-super open and fuzzy rg-closed, therefore $f^{-1}(G)$ is a proper fuzzy set of $X$, which is both fuzzy rg-super open and fuzzy rg-closed, because $f$ is fuzzy $\mathrm{rg}$-super continuous surjection. Therefore $\mathrm{X}$ is not fuzzy rgconnected, which is a contradiction. Hence $\mathrm{Y}$ is fuzzy rg- super connected.

\section{References}

[1]. B. Ghosh, Semi-continuous and semi-closed mappings and semi-connectedness in fuzzy setting,Fuzzy Sets and Systems 35(3) (1990), 345-355.

[2]. C. L. Chang, Fuzzy topological spaces, J. Math. Anal. Appl. 24 (1968), 182-190.

[3]. C.W. Baker on Preserving g-super closed sets Kyungpook Math. J. 36(1996), 195-199.

[4]. G. Balasubramanian and P. Sundaram, On some generalizations of fuzzy continuous functions,Fuzzy Sets and Systems 86(1) (1997), 93-100.

[5]. G. Balasubramanian and V. Chandrasekar, Totally fuzzy semi continuous functions, Bull. CalcuttaMath. Soc. 92(4) (2000), 305312.

[6]. G. Balasubramanian, On fuzzy pre-separation axioms, Bull. Calcutta Math. Soc. 90(6) (1998),427-434.

[7]. K. K. Azad, On fuzzy semi continuity, fuzzy almost continuity and fuzzy weakly continuity, J. Math. Anal. Appl. 82(1) (1981), 1432.

[8]. K. M. Abd El-Hakeim, Generalized semi-continuous mappings in fuzzy topological spaces, J. Fuzzy Math. 7(3) (1999), 577-589.

[9]. L. A. Zadeh, Fuzzy sets, Information and Control 8 (1965), 338-353.

[10]. M.K. Mishra et all on “Fuzzy super continuity” International Review in Fuzzy Mathematics vol-2,No-3,July -December2012,143146.

[11]. M.K. Mishra M. Shukla M. Fuzzy Regular Generalized Super Closed Set” International Journal of Scientific and Research December issue July December 2012 
[12]. M.K. Mishra,et all on " Fuzzy super closed set" International Journal International Journal of Mathematics and applied Statistics vol-3(I). ,43-46 July December 2012.

[13]. P. M. Pu and Y. M. Liu Fuzzy topology I Neighborhood structure of a fuzzy point and More-Smith Convergence. J. Math. Anal. Appl. 76(1980), 571-594.

[14]. P. M. Pu and Y. M. Liu Fuzzy topology II Product and quotient spaces J.Math. Anal. Appl. 77(1980) 20-37.

[15]. P. M. Pu, and Y. M. Liu, Fuzzy topology. I. Neighborhood structure of a fuzzy point and Moore-Smith convergence, J. Math. Anal. Appl. 76(2) (1980), 571-599.

[16]. R. K. Saraf and M. Khanna, On gs-closed sets in fuzzy topology, J. Indian Acad. Math. 25(1),(2003), 133-143.

[17]. R. K. Saraf, and M. Khanna, Fuzzy generalized semipreclosed sets, J. Tripura Math. Soc.3(2001) 59-68.

[18]. R. K. Saraf, and S. Mishra, Fg_-closed sets, J. Tripura Math. Soc. 2 (2000) 27-32.

[19]. R. K. Saraf, M. Caldas and S. Mishra, Results via Fg_-closed sets and Fg-closed sets, Pre print.

[20]. R. Malviya on certain concepts in fuzzy topology. Ph.D. Dissertation, R.D.V.V. Jabalpur (1997)

[21]. R. Prasad, S. S. Thakur and R. K. Saraf, Fuzzy rg-irresolute mappings, J. Fuzzy Math. 2(2)(1994), 335-339.

[22]. S. Bin Shahna, On fuzzy strong semi continuity and fuzzy pre continuity, Fuzzy Sets and

[23]. S. S. Thakur and S. Singh , Fuzzy M-semi pre continuous mappings, J. Indian Acad. Math.21(1)(1999), 39-44. 